\title{
Solvent-Induced Phase-Inversion and Electrical Actuation of Dielectric Copolymer Films
}

\author{
Yeonju Jang, Toshihiro Hirai \\ Smart Materials Engineering, Faculty of Textile and Technology, Shinshu University, Nagano, Japan. \\ Email: tohirai@shinshu-u.ac.jp
}

Received January $24^{\text {th }}, 2011$; revised February $8^{\text {th }}, 2011$; accepted February $11^{\text {th }}, 2011$.

\begin{abstract}
Block copolymers posses inherently the ability of form a variety of phase-separated microdomain structures. The lengths of block segments and the selectivity of the solvent are primary factors affecting the resultant morphology. This paper investigated the effect of casting solvents on the morphologies and electrical actuation of poly(methyl methacrylate)-poly(n-butyl acrylate)-poly(methyl methacrylate) (PMMA-PnBA-PMMA) triblock copolymer films comprising PMMA hard segment and PnBA soft segment. Transmission electron microscopy and confocal laser scanning microscopy observation revealed that PMMA and PnBA segments were assembled into various micro- and nano-sized phase structures where either of them formed continuous phase. This implies that continous phase could be inversed by used casting solvents. Solvent-dependent phase morphologies had a significant effect on the electrical actuation results. Increase of the PnBA contents and the continuous phases of PnBA soft segments improved both of electrical actuation and dielectric constant, indicating that solvent-induced phase separation modulates the electrical actuation of dielectric films. The significance of the role of solvent selectivity and the major continuous phase of the polymer in defining the morphology and electrical actuation of the self-assembled block copolymer structure are discussed.
\end{abstract}

Keywords: Actuator, Acrylic Triblock Copolymers, Electrical Actuation, Dielectric Properties

\section{Introduction}

Dielectric elastomer actuators (DEAs) behave as actuators by changing their shape in response to electrical stimulation. Due to their very good electromechanical capabilities in terms of fast response, large deformation, reliability and relatively high efficiency, several actuation applications such as loud-speakers, pumps, bio-inspired robotic systems and artificial muscles have been proposed. [1-3]. There are several active materials for these applications: piezoceramic and magnetostrictive materials, shape memory alloys, ferroelectric polymers and electroactive celluloses. The elastomers are composed of block copolymers, which have both hard and soft segments [4-8]. In particular, triblock copolymers are widely known as hybrid polymeric materials having a soft and rubbery matrix with hard, glassy domains. Due to their potential to form self-assembled structures, triblock copolymers have been used as active materials. Films with various morphologies have been prepared by diverse techniques such as melting, casting from different solvents, and solvent casting using different copoly- mer compositions which is the most widely used technique [9]. The hard segment can increase the modulus, and this physical property is related to the ultimate actuation performance $[10,11]$.

To improve the overall electromechanical response of a dielectric elastomer, suitable compliant electrodes are usually based on carbon black or graphite powders, either dispersed in grease and polymer matrices or directly deposited as thin layers [12]. When an appropriate voltage is applied to a DEA, the very soft nature of both the dielectric elastomer and its electrodes allows for significant coupling of the mechanical properties of the system with the electrostatic interactions arising between the induced polarization charges and the supplied surface free charges. This actuation mechanism is the so-called 'Maxwell stress', as depicted in Scheme 1. A voltage (V) is applied across the film, which is sandwiched between two compliant electrodes. The electrostatic force of attraction between the oppositely charged electrodes initiates a Maxwell stress that compresses the film in the transverse $(z)$ direction $[13,14]$. Like charges along each film surface repel each other, resulting in further stretch- 


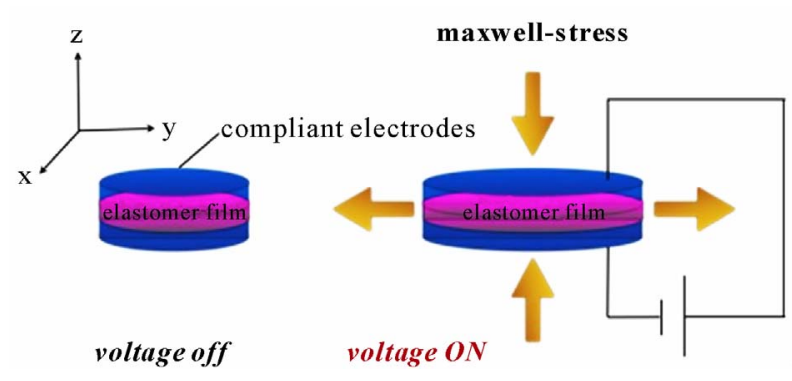

Scheme 1. Schematic illustration of a DEA, before (left) and after (right) electrical actuation. Electrostatic attraction between the oppositely charged compliant electrodes applied to opposing surfaces of the D-EAP generates a normal Maxwell stress upon actuation and compresses the film in the transverse (z) direction. Under isochoric conditions, the film extends laterally along the $x$ and $y$ directions. The thickness of the compliant electrodes is exaggerated here for clarity.

ing of the film along the lateral ( $x$ and $y$ ) directions. The effective electrostatic (Maxwell) stress is given [15] by $\varepsilon_{0} \varepsilon(V / t) 2$, where $\varepsilon_{0}$ is the permittivity of free space, $\varepsilon$ represents the field-invariant dielectric constant of the DEA film and $V / t$ is the electric field. The stress acting along the $z$ axis generates a transverse strain, as well as in-plane stresses along the $x$ and $y$ directions. The extent and distribution of the accompanying in-plane strains upon actuation are governed by the elastic modulus $(E)$ of the DEA and any in-plane anisotropy, which can be introduced by dissimilar directional pre-straining of the film $[16,17]$.

Other methods have been proposed recently to enhance the actuation performance. The most widely adopted methods are based on the production of composites, by loading an elastomer matrix with insulating or conductive fillers (either organic or inorganic). However, the fillers make the film stiffer, thus low stretchability is obtained $[18,19]$. To bypass such limitations, blending a dielectric elastomer with a highly polarizable phase, rather than loading it with filler, has been proposed more recently [20]. However, the contents of a highly polarizable phase are difficult to control for blend uniformity. For DEA films that have an irregular structure, sufficiently accurate values of the dielectric constant, which is one of the important factors determining actuation performance, are difficult to obtain [21]. To improve the actuation performance of a dielectric elastomer it is thus necessary to focus on those properties, namely the elastic modulus and dielectric constant, that directly control both stress and strain. In addition, investigation is needed of the phase morphology of DEA films, which influences deformation, elastic modulus and response to strain. When the hard segment make continuous phase, the film getting harder while the soft segment forms continuous phase, the flexible film is obtained.

In the present study a simple approach is proposed to make the films with controllable morphologies. New dielectric elastomer films with improved actuation performance were obtained by controlling the casting solvents without any additives. The systems that were studied were two poly(methyl methacrylate)-poly(n-butyl acrylate)-poly(methyl methacrylate)(PMMA-PnBA-PMMA) block copolymers with different composition. The formulated materials were then characterized in terms of their dielectric constant, tensile properties and electrical actuation. To clarify the relation between phase morphologies and physical properties, the phase structures of films were observed by transmission electron microscopy (TEM) and confocal laser scanning microscopy to find the morphologies both of micro- and nanosize.

\section{Experimental}

\subsection{Materials}

Poly(methyl methacrylate)- $b$-poly( $n$-butyl acrylate)- $b$-poly (methyl methacrylate) (PMMA-PnBA-PMMA) triblock copolymers with different block lengths were employed in conjunction with various solvents. Two PMMA-PnBAPMMA copolymers, supplied by Kuraray Co. Ltd., Japan, were synthesized by living anionic polymerization [22, 23] with 23\% PMMA (molecular weight $72000 \mathrm{~g} \cdot \mathrm{mol}^{-1}$ ) and $31 \%$ PMMA (molecular weight $132000 \mathrm{~g} \cdot \mathrm{mol}^{-1}$ ): the corresponding dielectric elastomer films are denoted TAPM23 and TCPM31, respectively. Toluene, chloroform, tetrahydrofuran and acetone (Wako Pure Chemical Industries, Ltd., Japan) were used as casting solvents to obtain phase inversion with different continuous phase either of PMMA or PnBA because of their different affinities. The three dimensional solubility parameters of the homopolymers (PMMA and PnBA) and the solvents are listed in Table 1. Graphite powder (99.9995\% metal basis, Alfa Aesar) and silicone grease (Shin Etsu Chemi-

Table 1. Solubility parameters of various solvents and polymers $d_{t}$ : total solubility parameter, $d_{d}$ : dispersive parameter, $d_{p}$ : polar parameter, $d_{h}$ : hydrogen bonding parameter.

\begin{tabular}{cccccc}
\hline Name & $\begin{array}{c}\text { Molecular } \\
\text { formula }\end{array}$ & $d_{t}$ & $d_{d}$ & $d_{p}$ & $d_{h}$ \\
\hline $\begin{array}{c}\text { Poly } \\
\text { (n-butylacrylate) }\end{array}$ & $\mathrm{C}_{7} \mathrm{H}_{12} \mathrm{O}_{2}$ & 10.18 & 16.38 & 6.63 & 5.77 \\
Toluene & $\mathrm{C}_{7} \mathrm{H}_{8}$ & 18.2 & 18.0 & 1.4 & 2.0 \\
Chloroform & $\mathrm{CHCl}_{3}$ & 18.84 & 17.69 & 3.07 & 5.73 \\
THF & $\mathrm{C}_{4} \mathrm{H}_{8} \mathrm{O}$ & 19.47 & 16.81 & 5.73 & 7.98 \\
$\begin{array}{c}\text { Acetone } \\
\begin{array}{c}\text { Poly } \\
\text { (methylmethacrylate) }\end{array}\end{array}$ & $\mathrm{CH}_{3} \mathrm{COCH}_{3}$ & 20.1 & 15.5 & 10.4 & 7.0 \\
\hline
\end{tabular}


cal Co., Ltd.) were used as the compliant electrode materials in the actuation experiment.

\subsection{Preparation of PMMA-PnBA-PMMA Triblock Copolymer Films}

The general procedures for preparing dielectric thin films were identical in each case, but the triblock copolymer and solvent were varied. The PMMA-PnBA-PMMA triblock copolymer films were cast from $6.25 \%(\mathrm{w} / \mathrm{v})$ each of solvents for 1 day; then the solution was transferred to a PTFE laboratory dish at room temperature. After 7 days of solvent evaporation, transparent circular films with radius $8 \mathrm{~cm}$ and nominal thickness $100 \mu \mathrm{m}$ were formed (Figure 1).

\subsection{Dielectric Characterization}

Electromechanical impedance measurement was used to determine the dielectric constant of the fabricated films. The apparatus used for the measurements was a Solarton Co. model 1250 frequency response analyzer coupled with a model 1286 potentiostat via an IEEE interface (National Instruments, Austin, TX) to a 586 PC (Solartron software Zplot and Zview). Impedance measurement was performed in the frequency range 0 to $25000 \mathrm{~Hz}$ with signal amplitude $100 \mathrm{mV}$. A Pt plate electrode is used as counter electrode, and $\mathrm{Ag} / \mathrm{AgCl}$ as reference electrode. Circular disk-shaped specimens $10 \mathrm{~mm}$ in diameter and $1 \mathrm{~mm}$ thick were used.

\subsection{Morphology Observation}

The nanoscale phase-separated morphologies of films were observed with a transmission electron microscope (Hitachi High-Technologies Corporation. model H-7100 FA). The micrographs were obtained using $100 \mathrm{kV}$ acceleration voltage. The sample films were prepared in ultra-thin sections by means of a cryo-ultra microtome, and the PMMA phase was selectively stained with phos-

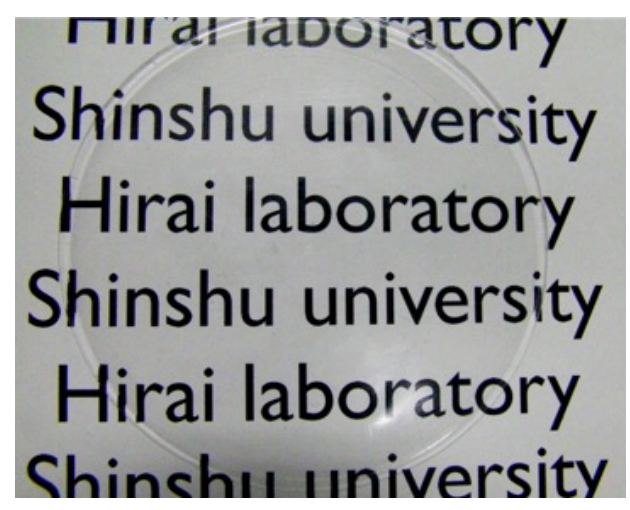

Figure 1. Fabricated dielectric thin film. The transparent circular film has a radius of $8 \mathrm{~cm}$ and nominal thickness of $100 \mu \mathrm{m}$ was formed. photungstic acid (PTA). The microscale phase-separated surface was examined using a confocal laser scanning microscope (LEXT OLS3100, Olympus Co., Japan). Confocal images were obtained using a x100 objective with a $408 \mathrm{~nm}$ incident laser beam.

\subsection{Mechanical Characterization}

Measurements were performed using a Tensilon mechanical testing machine (RTC tensile tester from A\&D Co. Ltd). Sample films with standard size [ISO 37-2 (JIS $\mathrm{K}$ 6251-6)] were drawn at $25 \mathrm{~mm} \cdot \mathrm{min}$ at $20^{\circ} \mathrm{C}$. The variation of tensile stress with draw ratio was recorded. Elastic modulus, elongation at break, and stress at break were determined as averages of five independent experiments performed under the same conditions.

\subsection{Electrical Actuation}

Electrical actuation was conducted with a circular actuator (radius $\mathrm{R}=50 \mathrm{~mm}$ ). To make an actuator, fabricated films were $100 \%$ biaxially pre-strained with a custommade tensile jig designed to reduce the thickness, and therefore the voltage level required for actuation. Generally, dielectric elastomer films are routinely prestrained uniaxially to improve their actuation performance [17, 24-27]. Pre-straining tends to reduce the dielectric constant while simultaneously enhancing the dielectric breakdown strength [25-27]. Mechanically compliant electrodes (a mixture of graphite powder and silicone grease) were deposited on both sides of the film, leaving the border area free of conduction paths to avoid possible arcing (electrical short). The two electrodes were connected to a high voltage supply by means of thin aluminum tape. The effects of the application of an electric field were investigated by increasing the voltage in steps of 500 voltages. A video recorder was used to record the circular actuation behavior of the films, and the nominal radial strain of the coated area was measured and analyzed using the ImageJ image analysis program.

\section{Results and Discussion}

\subsection{Dielectric Characterization}

To elucidate the general response of TAPM23 and TCPM31 films under loading during actuation, various electric field strengths were used. The relative dielectric constant was measured for all of the formulations. Selected results for various systems are shown in Figures 2 and 3. The dielectric constant of PMMA film, a dielectric polymer, was measured as a control and compared with those of the copolymer films. Unlike the other fabricated dielectric films, PMMA film was measured with no pre-strain, because attempts to strain PMMA film to the same extent caused the film to break due to its fragility. 


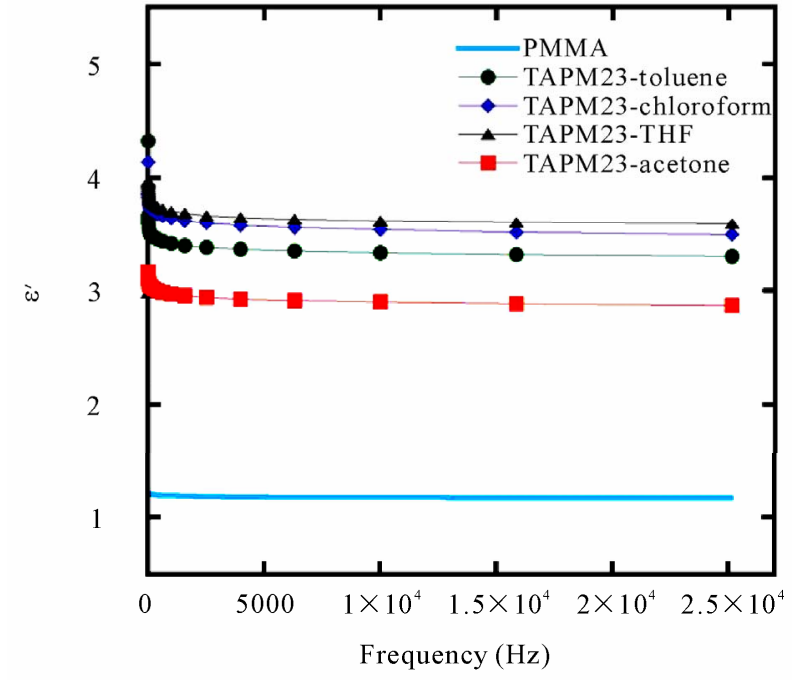

Figure 2. Relative dielectric constant as a function of frequency for TAPM23 films with a various casting solvents. The solid line identifies dielectric constant of PMMA film.

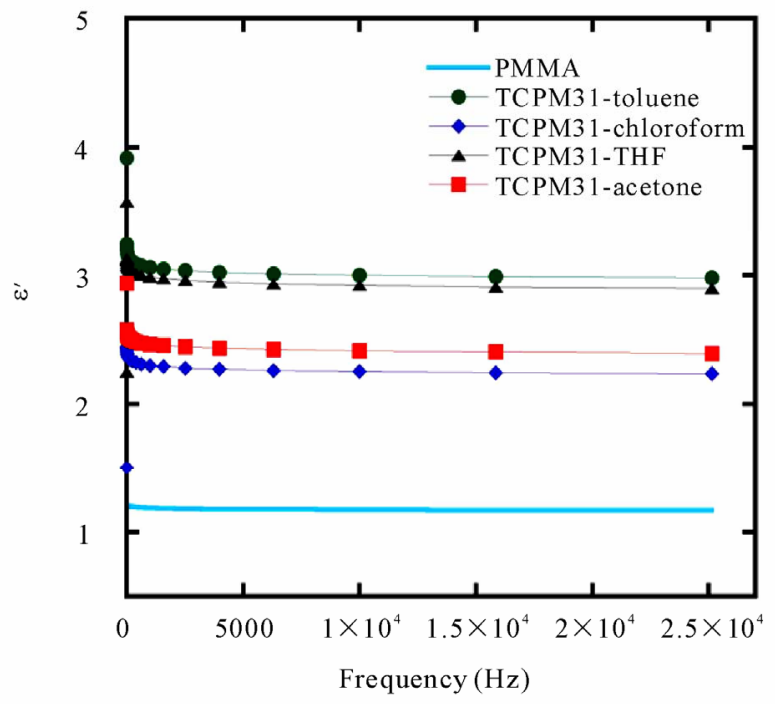

Figure 3. Relative dielectric constant as a function of frequency for TCPM31 films with a various casting solvents. The solid line identifies dielectric constant of PMMA film.

The dielectric constants of the TAPM23 films were higher than the values for the TCPM31 films overall; the observed difference depended on molecular weight and composition of the triblock copolymers (the molecular weight of TCPM31 was twice that of TAPM23). As a general rule, solubility decreases as the molar mass of the solute increases [28]. As electrical forces due to polarizability and polar moment determine the cohesive energy, a correlation between dielectric constant and solubility parameter may be expected [29]. Interestingly, both TAPM23 film and TCPM31 film had very low values of the dielectric constant when acetone was used as casting solvent. It appears that when acetone was used, the polymer film was formed with PMMA as the continuous phase due to the close affinity of acetone and PMMA (Table 1).

Another important observation can be made in relation to the spectra of Figure 2 and Figure 3. The films made from TAPM23 showed higher dielectric constants overall except for those made in acetone. This behavior is related to the solubility parameters shown in Table $\mathbf{1}$. The solubility parameter of acetone is much closer to that of PMMA than to the value for PnBA. Considering that acetone has a weak dispersive contribution, the other components (the polar component and the hydrogen bonding component) have a strong effect in the polymer solutions. We recognize also that to achieve good dispersion, the polar component of the solubility parameter is important, while the other components have been reported by Jing et al. [30]. This corresponds to the dielectric constant results for the fabricated films. The details of the relationship of the solubility parameter to the dielectric constant of the polymer are considered in the morphology section. The dielectric constant results for the TCPM31 films showed a similar trend to those of TAPM23 films (Figure 2), which suggests that the solubility parameter has a non-negligible effect. However, the TCPM31 film cast from chloroform showed anomalous behavior, which can be related to the electrical actuation results shown in Figure 8. The TCPM31 film cast from chloroform had the smallest dielectric constant (Figure 3) and a corresponding small nominal radial strain (\%) in its electrical actuation result (Figure 8). Zhang and coworkers have proposed an elastic energy density to measure both the stress and strain generation capability of an actuator material [31]. The energy density is $U_{E}=1 / 2 K \varepsilon_{0} E^{2}$ where $E$ is the applied field, $\varepsilon_{0}$ is the vacuum dielectric constant $\left(=8.85 \times 10^{-12} \mathrm{~F} / \mathrm{m}\right)$, and $K$ is the dielectric constant of the polymer. The dielectric constants of all fabricated dielectric films correspond to the electrical actuation results of those films.

\subsection{Mechanical Properties of PMMA-PnBA-PMMA Films}

Figures 4 and 5 show the stress-strain responses of TAPM23 films and TCPM31 films, respectively. All of the curves show an up-swing at high elongations. However, a marked reduction of the rupture point was observed for the TAPM23 film that was cast from acetone. Such behavior can be ascribed to the affinity of the acetone casting solvent for the PMMA blocks in the triblock copolymers. The other TAPM23 films retained the up-swing at high elongations, indicative of close affinity 


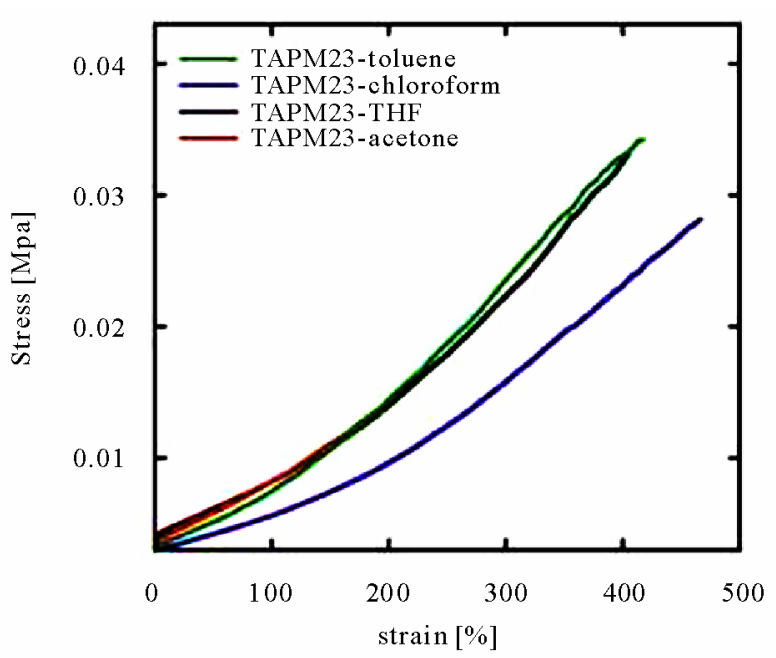

Figure 4. Stress-strain tensile mechanical properties for TAPM23 films with a various casting solvents.

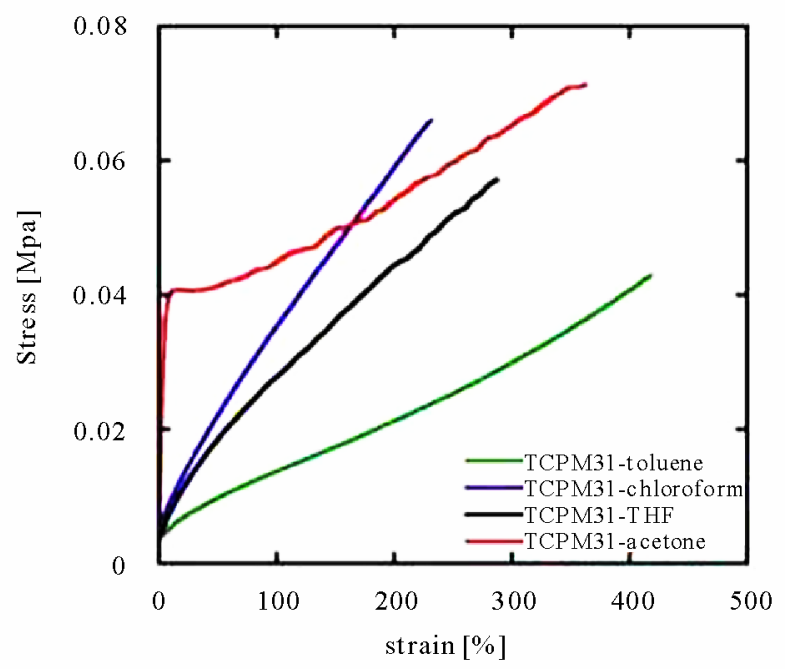

Figure 5. Stress-strain tensile mechanical properties for TCPM31 films with a various casting solvents.

between PnBA blocks and their casting solvents (Table 1).

Markedly different mechanical properties were found for the TCPM31 film cast from acetone compared to those of the other TCPM31 films of Figure 5. The stressstrain curve of the TCPM31 film cast from acetone reflects hard and tough polymer characteristics, which are intimately related to the lamellar phase morphology of that film (Figure 6(h)) [32]. The mechanical response of the TCPM31 films for various solvents can be ascribed to their polymer-solvent affinities and correlated with their phase morphologies. The tensile stress-strain curves of TCPM31 films cast from toluene, chloroform and tetrahydrofuran, which have close affinity to the PnBA phase, show soft property behavior. In the case of TCPM31

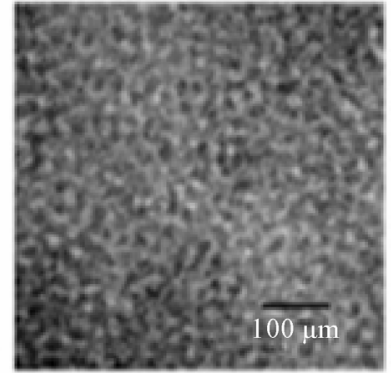

TAPM23-THF

(a)

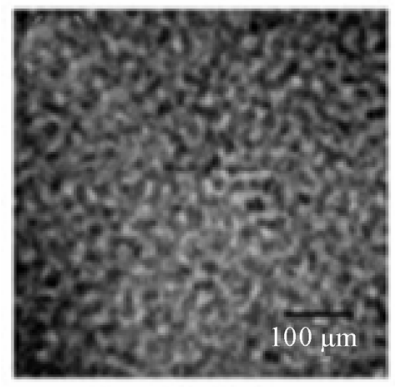

TAPM23-toluene

(c)

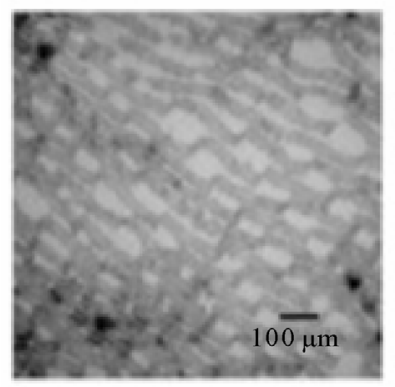

TCPM31-chloroform

(e)

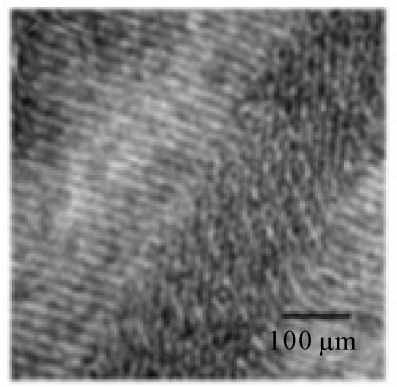

TCPM31-THF

(g)

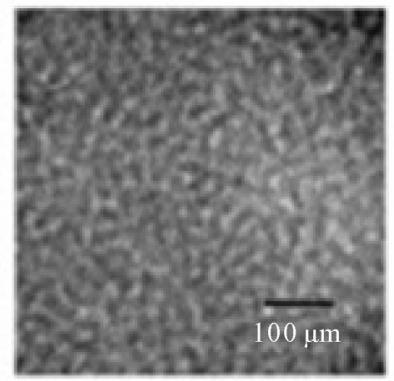

TAPM23-chloroform

(b)

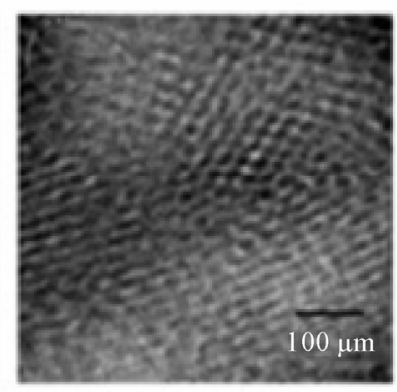

TAPM23-acetone

(d)

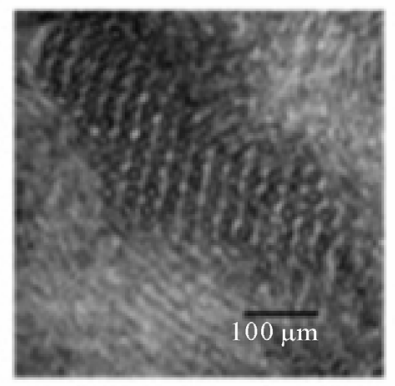

TCPM31-toluene

(f)

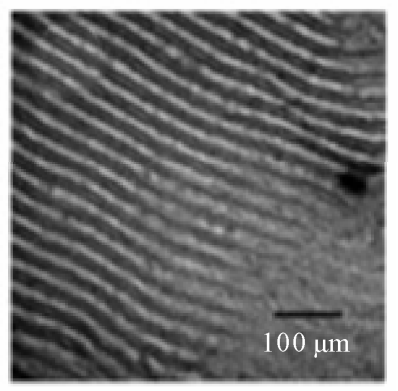

TCPM31-acetone

(h)
Figure 6. TEM photographs of ultra-thin cross-sections of triblock copolymer films. The bright regions represent the PnBA phase and the dark regions correspond to the PMMA phase. Top line (a-d) show the TAPM23 films cast from THF, chloroform, toluene and acetone, respectively. Bottom line (e-h) represent to the TCPM31 films cast from chloroform, toluene, THF and acetone, respectively. 
films cast from toluene and tetrahydrofuran, the stressstrain curves showed a similar trend to cylindrical morphology, though lamellar phase morphology is included in their structure. It appears that the cylindrical morphology is generated in preference to lamellar morphology when they coexist. Moreover, the degree of alignment of the lamellar structure has an effect on their mechanical properties. The TCPM31 film cast from tetrahydrofuran, which had a more ordered lamellar structure, was stiffer than the TCPM31 film cast from toluene.

\subsection{Morphologies of PMMA-PnBA-PMMA Films}

Because of the incompatibility between PnBA and PMMA, the triblock copolymers were expected to show phase separation. To visualize this effect, TEM imaging was carried out on thin films prepared in ultrathin sections by means of acryo-ultra microtome, and the PMMA phase was selectively stained with phosphotungstic acid (PTA). The various phase structures were obtained for each of films and are shown in Figures 6(a-h). From the viewpoint of comparing TAPM23 and TCPM31 films, increasing the molecular weight of the triblock copolymers led to more oriented phase morphology. The morphology of TAPM23 copolymer films with PMMA content 23\%, cast from various solvents, showed PnBA cylinders dispersed in the PMMA phase.

On the other hand, the TCPM31 copolymer with $69 \%$ of PnBA showed a more oriented cylindrical morphology, excluding the TCPM31 film cast from acetone. This difference of film microstructure arose from the affinity between polymer segments and solvent, because the microstructure is strongly influence by interfacial interactions at the polymer-solvent interface $[33,34]$. The casting solvents used in this study, excluding acetone, had higher affinity to PnBA segments than to PMMA segments (Table 1). It can be inferred that the preferential interaction of the solvent to one of the blocks in block copolymers has a strong effect on self-assembly in films of the block copolymers. The micro-phase separated structure is due to the incompatibility between the different connected block chains. The compatibility of solvent and block copolymer is a function of the interaction parameter $\left(X_{A B}\right)$ between the component polymers $\mathrm{A}$ and B. The interaction parameter; $X_{A B}$ is related to the solubility parameters by the relation $[35,36]$.

$$
X_{A B}=\operatorname{Vr} / R T\left(\delta_{A}-\delta_{B}\right)^{2}
$$

where $V r$ is the molar volume of the smaller repeat unit, $R$ the universal gas constant, $T$ absolute temperature, and $\delta_{A}$ and $\delta_{B}$ are the solubility parameters of polymers $A$ and $B$, respectively. The expression for $\delta$ includes contribu- tions from dispersive forces $\left(\delta_{d}\right)$, dipole/dipole $\left(\delta_{p}\right)$ and hydrogen bonding $\left(\delta_{h}\right)$ interactions. The total solubility parameter $\delta_{t}$ is related to these individual components by

$$
\delta_{t}=\delta_{d}^{2}+\delta_{p}^{2}+\delta_{h}^{2}
$$

The morphology of TCPM31 copolymer film cast from acetone has a close affinity with PMMA than PnBA (Table 1). For this reason, the phase structure of this film showed the lamellar structure with a continuous phase of PMMA. This ordered structure was observed on micro scale by confocal laser scanning microscopy as shown in Table 2. All of the points had nearly equal separation of on average of $0.6 \mu \mathrm{m}$. A similar pattern for the lamellar structure means that the structure was regularly ordered.

Anomalous phase morphology was observed for TCPM31 film cast from chloroform. To the best of our knowledge, non-equilibrium structure have trapped during the progress of the coexitence phases with cylindrical and lamellar morphologies like the phase morphology of TCPM31, made from tetrahydrofuran because tetrahydrofuran and chloroform are easy to quickly vaporize. This anomalous phase morphology is reflected in the anomalous performance of the film in the electrical actuation experiment. Comparison of the TEM images for TCPM31 films cast from tetrahydrofuran and toluene shows that the film cast from tetrahydrofuran had a more ordered lamellar structure with cylindrical morphology than the TCPM31 film cast from toluene, which is consistent with the respective electrical actuation results. We conclude that the phase morphologies are significantly associated with the electrical actuation results.

\subsection{Electrical Actuation of PMMA-PnBA-PMMA Films}

To explore the relationship between phase morphology and actuation behavior of the films, the responses of circular actuators, made from TAPM23 and TCPM31 copolymers with various casting solvents, to electric fields

Table 2. Confocal laser scanning microscope image and the distances of phases of TCPM31 film cast from acetone.

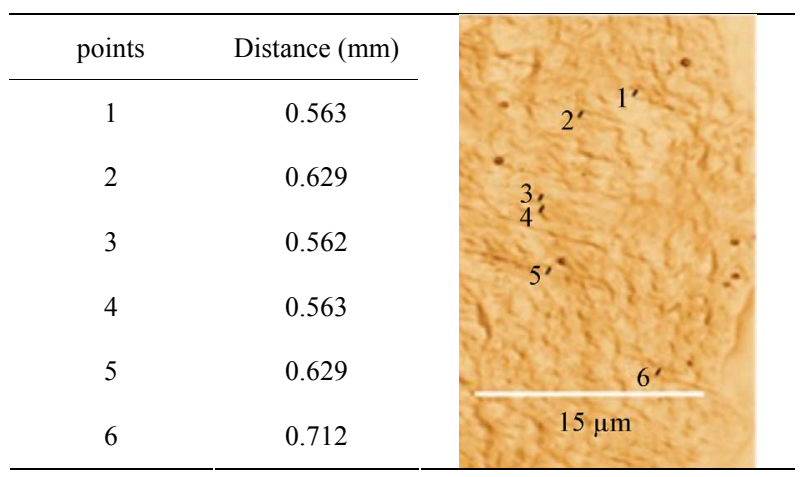


were observed and measured. Pre-strained films were fixed into the circular frame, and the circular center of the films was coated with compliant electrodes to form an electrical contact on both sides of the films. When a voltage was applied across the film, the electrostatic force of attraction between the oppositely charged electrodes initiated a Maxwell stress that compressed the film. The results of nominal radial strain (\%) of TAPM23 and TCPM31 films are presented in Figures 7 and 8, respecttively. The TAPM23 films had greater deflections than those of TCPM31 films. The films that formed a PnBA continuous phase with cylindrical structure showed large (almost 14\%) nominal radial strain and reproduceble electrical actuation behavior was observed. Moreover, the films started to actuate at a lower critical electric field strength. However, the TAPM23 film cast from acetone reached dielectric breakdown at lower electric fields. This behavior can be ascribed to the low dielectric constant of that film, compared with other films, and the highly ordered phase morphology. The structure formed with PMMA continuous phase can be a non-negligible reason.

Interesting performance was shown by the TCPM31 films (Figure 8). In the cases of films with lamellar microstructure, the deformation of the films exhibited a stepwise response with increasing electric field strength. However, the TCPM31 film cast from toluene had a less ordered lamellar structure, hence the deformation showed an almost quadratic relationship with the electric field. The presence of the ordered lamellar PMMA domains significantly obstructed the actuation [36]. Overall, the TCPM31 film was in a stable state in high electric fields, whereas the nominal radial strain (\%) was small.

\section{Conclusions}

Relevant properties of two triblock copolymers with dif-

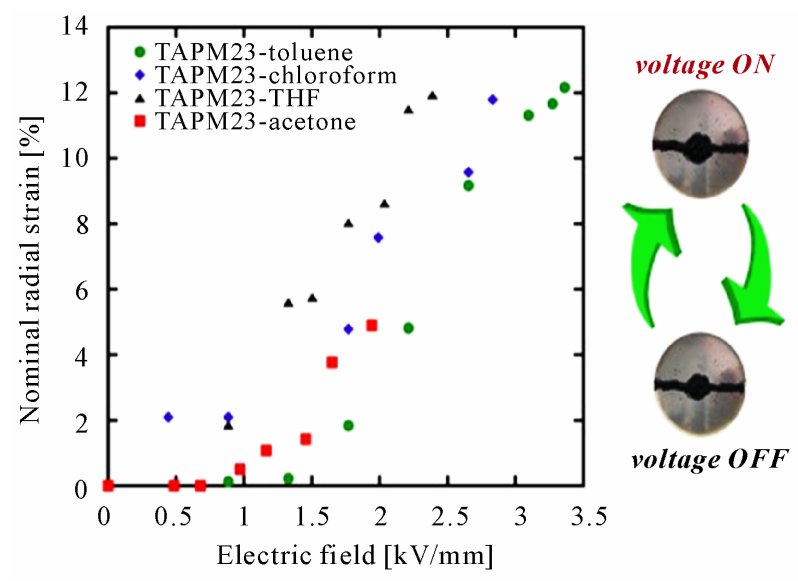

Figure 7. Electrical actuation results for TAPM23 films with various casting solvents.

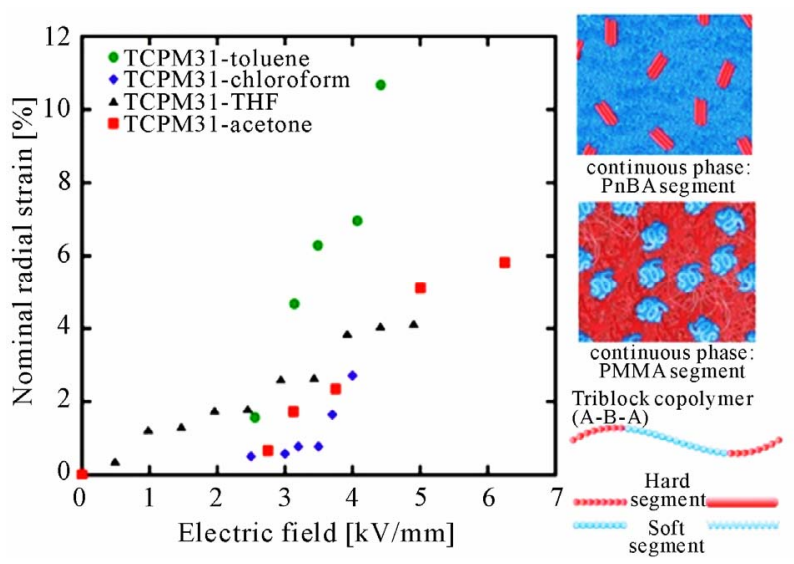

Figure 8. Electrical actuation results for TCPM31 films with various casting solvents.

ferent molecular weights, prepared by casting from various solvents, were studied and compared by examining the dielectric constant and electrical actuation. For the PMMA-PnBA-PMMA triblock copolymer films, the electrical actuation results were consistent with the dielectric constants and phase morphology and the tensile stress-strain data. Smaller content of hard segments and the soft segment as a continuous phase made it possible to obtain remarkable performance in their actuation behaviors by increasing the dielectric constant.

Further study is in progress, with the objective of better clarifying the influence of various soft segments and soft segment contents on the electromechanical performance.

\section{Acknowledgements}

This work is supported by a Grant-in-Aid for Global COE program by the Ministry of Education, Culture, Sports, Science, and Technology. We thank the Kuraray Co. Ltd., for providing materials ans we thank Syuji Kobukata for his support for the TEM analysis.

\section{REFERENCES}

[1] R. Kornbluh, R. Pelrine, Q. Pei, S. Oh and J. Joseph, "Ultrahigh Strain Response of Field-Actuated Elastomeric Polymers," Proceedings of SPIE, 2002, pp. 51-64.

[2] R. Pelrine, R. Kornbluh, Q. Pei and J. Joseph, "Highspeed Electrically Actuated Elastomers with over 100\% Strain," Science, Vol. 287, No. 5454, 2000, pp. 836-839. doi:10.1126/science. 287.5454 .836

[3] P. Carpi, D. de Rossi, R. Kornbluh, R. Pelrine and P. Sommer-Larsen, "Dielectric Elastomers as Electromechanical Transducers: Fundamentals, Materials, Devices, Models and Applications of an Emerging Electroactive Polymer Technology," Elsevier, Amsterdam, 2008.

[4] L. E. Cross, "Ferroelectric Ceramics: Materials and Application Issues," Ceramic Transactions, Vol. 68, 1996, 
pp. $15-55$.

[5] K. B. Hathaway and A. E. Clark, "Magnetostrictive Materials," MRS Bulletin, Vol. 18, No. 4, 1993, pp. 34-41.

[6] S. M. Wayman, "Shape Memory Alloys," MRS Bulletin, Vol. 18, No. 4, 1993, pp. 49-56.

[7] A. J. Lovinger, "Ferroelectric Polymers," Science, Vol. 220, No. 4602, 1983, pp. 1115-1121. doi:10.1126/science.220.4602.1115

[8] J. Kim, Y. Kang and S. Yun, "Force Measurement of Electro-Active Paper Actuators by Microbalance," Sensors and Actuators A: Physical, Vol. 133, No. 2, 2007, pp. 401-406. doi:10.1016/j.sna.2006.04.019

[9] H. H. Winter, D. B. Scott, W. Gronski, S. Okamoto and T. Hashimoto, "Ordering by Flow near the Disorder-Order Transition of Triblock Copolymer Styrene-Isoprene-Styrene," Macromolecules, Vol. 26, No. 26, 1993, pp. 72367244. doi:10.1021/ma00078a019

[10] Y. Miura, T. Kaneko, K. Satoh, K. Kamigaito, H. Jinnai and Y. Okamoto, " $\mathrm{A}_{\mathrm{x}} \mathrm{BA}_{\mathrm{x}}$-Type Block-Graft Polymers with Soft Methacrylate Middle Segments and Hard Styrene Outer Grafts: Synthesis, Morphology, and Mechanical Properties," Asian Journal of Chemistry, Vol. 2, No. 5, 2007, pp. 662-672. doi:10.1002/asia.200600403

[11] W.-K. Lee, H.-D. Kim and E.-Y. Kim, "Morphological Reorientation by Extensional Flow Deformation of a Triblock Copolymer Styrene-Isoprene-Styrene," Current Applied Physics, Vol. 6, No. 4, 2006, pp. 718-722. doi:10.1016/j.cap.2005.04.026

[12] R. Shankar, T. K. Ghosh and R. J. Spontak, "Mechanical and Actuation behavior of Electroactive Nanostructured Polymers," Sensors and Actuators A: Physical, Vol. 151, No. 1, 2009, pp. 46-52. doi:10.1016/j.sna.2009.01.002

[13] R. Shankar, T. K. Ghosh and R. J. Spontak, "Electroactive Nanostructured Polymers as Tunable Actuators," Advanced Materials, Vol. 19, No. 17, 2007, pp. 22182223. doi:10.1002/adma.200602644

[14] R. Pelrine, R. Kornbluh and G. Kofod, "High-Strain Actuator Materials Based on Dielectric Elastomers," $A d$ vanced Materials, Vol. 12, No. 16, 2000, pp. 1223-1225. doi:10.1002/1521-4095(200008)12:16<1223::AID-ADM A1223>3.0.CO;2-2

[15] R. Kornbluh, R. Pelrine, J. Joseph, R. Heydt, Q. Pei and S. Chiba, "High-Field Electrostriction of Elastomeric Polymer Dielectrics for Actuation," Proceeding of SPIE, Vol. 3669, 1999, pp. 149-161. doi:10.1117/12.349672

[16] G. Kofod, R. Kornbluh, R. Pelrine and P. Sommer-Larsen, "Actuation Response of Polyacrylate Dielectric Elastomers," Proceeding of SPIE, Vol. 4329, 2001, pp. 141-147. doi:10.1117/12.432638

[17] G. Kofod, P. Sommer-Larsen, R. Kornbluh and R. Pelrine, "Actuation Response of Polyacrylate Dielectric Elastomers," Journal of Intelligent Material Systems and Structures, Vol. 14, No. 12, 2003, pp. 787-793. doi: $10.1177 / 104538903039260$

[18] G. Gallone, F. Carpi, D. de Rossi, G. Levita and A. Marchetti, "Dielectric Constant Enhancement in a Sili- cone Elastomer Filled with Lead Magnesium NiobateLead Titanate," Materials Science and Engineering: C, Vol. 27, No. 1, 2007, pp. 110-116. doi:10.1016/i.msec.2006.03.003

[19] F. Carpi and D. de Rossi, "Improvement of Electromechanical Actuating Performances of a Silicone Dielectric Elastomer Dispersion of Titanium Dioxde Powder," IEEE Transactions on Dielectrics and Electrical Insulation, Vol. 12, No. 4, 2005, pp. 835-843. doi:10.1109/TDEI.2005.1511110

[20] A. J. Moulson and J. M. Herbert, "Electroceramics," Chapman \& Hall, London, 1990.

[21] G. Gallone, F. Galantini and F. Carpi, "Perspectives for New Dielectric Elastomers with Improved Electromechanical Actuation Performance: Composites versus Blends," Polymer International, Vol. 59, No. 3, 2010, pp. 400-406. doi:10.1002/pi.2765

[22] K. Ishiura, “接着の技術 (Settyaku No Gijutsu),” Vol. 25, 2005, pp. 38-43.

[23] Y. Morishita, T. Kurihara, K. Hamada and K. Ishiura, “A Novel (Meth)Acrylic Block Co-Polymers Synthesis, Characteristics, and Applications," The 8th SPSJ International Polymer Conference, 28P2G5-102b, 2005, p. 573.

[24] R. Palakodeti and M. R. Kessler, "Influence of Frequency and Prestrain on the Mechanical Efficiency of Dielectric Electroactive Polymer Actuators," Materials Letters, Vol. 60, No. 29-30, 2006, pp. 3437-3440. doi:10.1016/j.matlet.2006.03.053

[25] H. R. Choi, K. Jung, N. H. Chuc, M. Jung, I. Koo, J. Lee, J. Nam and M. Cho, "Effects of Prestrain on Behavior of Dielectric Elastomer Actuator," Proceeding of SPIE, Vol. 5759, 2005, pp. 283-291. doi:10.1117/12.599363

[26] X. Zhang, M. Wissler, B. Jaehne, R. Breonnimann and G. Kovacs, "Eletroactive Polymer Actuators and Devices (EAPAD)," Smart Structures and Materials: SPIE, Vol. 5385, 2004, pp. 78-86.

[27] A. O'Halloran and F. O'Malley, "Materials and Technologies for Artificial Muscle: A Review for the Mechatronic Muscle Project," In: P. J. Prendergast and P. E. McHugh, Eds., Topics in Bio-Mechanical Engineering, Trinity Centre for Bioengineering \& National Centre for Biomedical Engineering Science, 2004, pp. 184-215.

[28] D. W. van Krevelen and K. te Nijenhuis, "Properties of Prolymers," 4th Edition, Elsevier, Oxford, Chap. 7, 2008.

[29] D. W. van Krevelen and K. te Nijenhuis, "Properties of Prolymers," 4th Edition, Elsevier, Oxford, Chap. 11, 2008.

[30] J. Liu, T. Liu and S. Kumar, "Effect of Solvent Solubility Parameter on SWNT Dispersion in PMMA," Polymer, Vol. 46, No. 10, 2005, pp. 3419-3424. doi:10.1016/j.polymer.2005.02.086

[31] Q. M. Zhang, H. Li, M. Poh, F. Xia, Z. Y. Cheng, H. Xu and C. Huang, "An All-Organic Composite Actuator Material with a High Dielectric Constant," Nature, Vol. 19, No. 419, 2002, pp. 284-287. doi:10.1038/nature01021

[32] S. Ashley, "Artificial Muscles," Scientific American, Vol. 289, No. 4, October 2003, pp. 52-59. 
doi:10.1038/scientificamerican1003-52

[33] M. E. Seitz, W. R. Burghardt, K. T. Faber and K. R. Shull, "Self-Assembly and Stress Relaxation in Acrylic Triblock Copolymer Gels," Macromolecules, Vol. 40, No. 4, 2007, pp. 1218-1226. doi:10.1021/ma061993+

[34] R. D. Deanin, "Encyclopedia of Polymer Science and Technology, Wiley, New York, Chap. 7, 1977.

[35] H. Burrel, "Polymer Handbook," Interscience, New York,
1975.

[36] K. Thongsak, R. Kunanuruksapong, A. Sirivat and W. Lerdwijitjarud, "Electroactive Styrene-Isoprene-Stryene Triblock Copolymer: Effects of Morphology and Electric Field," Materials Science and Engineering: A, Vol. 527, No. 10-11, 2010, pp. 2504-2509. doi:10.1016/j.msea.2010.01.036 